\title{
Diagnosis of Carotid Artery Atheroma by Magnetic Resonance Imaging
}

\author{
Katsuhiro Ono, MD; Shigeru Watanabe, MD; Yumiko Daimon, MD; \\ Hideki Sakurada, MD; Miharu Urano, MD; Kezhu Sun, MD; \\ Yasuyoshi Hijikata, MD; Toshihisa Inoue, MD; Yoshiaki Masuda, MD
}

\begin{abstract}
Atheroma appears as a very low signal intensity area on 2-dimensional time-of-flight (TOF) magnetic resonance (MR) images, and its components have various signal intensities on spin-echo (SE) images. The present study investigated atheroma of the carotid arteries in 37 subjects with risk factors ( $63 \pm 10$ years of age; 19 men) by magnetic resonance imaging (MRI). On 2-dimensional (2D) TOF images, the carotid arteries were clearly demonstrated in all cases and atheroma was detected in 23 patients. The most common location of atheroma was at the origin of the internal carotid artery. There was vascular remodeling in all patients with atheroma. 2D-TOF images showed $97 \%$ agreement with ultrasonography. SE images clearly demonstrated atheroma in all 23 patients with atheroma. All patients with atheroma showing high signal intensity on T1-weighted images had hyperlipidemia. These findings indicate that the 2D-TOF imaging method is useful for detecting atheroma and SE-images are useful for its characterization. (Jpn Circ J 2001; 65: 139-144)
\end{abstract}

Key Words: Atheroma; Lipid component; Magnetic resonance image; Spin echo; Time-of-flight

$A$

herosclerosis of the carotid artery is the primary cause of stroke, and cerebral infarction is the major cause of death. Infarction occurs secondary to plaque ulceration, thrombosis, and intraplaque hemorrhage ${ }^{1-3}$ The stability of atheroma has been investigated by angiography, ultrasound, and magnetic resonance angiography (MRA) and of these, angiography is the standard imaging technique for diagnosing arterial stenosis. However, not only the extent of stenosis but also the features of the atheroma are important in determining the risk of clinical events. Some atheromatous plaques are stable, whereas others are fragile. Because emboli from atheroma are involved in the pathogenesis of transient ischemic attacks, changes in the components of atheroma are also important. The gold standard for determining these features of atheroma is still histological examination.

We have been investigating noninvasive techniques for the detection of atheroma and MRA is a noninvasive alternative to conventional angiography, 4 With respect to magnetic resonance imaging (MRI), it has been reported that intraluminal lesions appear as a signal void within the high signal intensity blood on 2-dimensional time-of-flight (2D-TOF) images, ${ }^{6}$ whereas the components of the atheroma can be detected on spin-echo (SE) images?-9 In the present study, we attempted to detect atheroma of the carotid arteries by 2D-TOF and investigated whether the features of atheroma had any relation to cardiovascular risk factors.

(Received September 4, 2000; revised manuscript received November 9 , 2000; accepted November 21, 2000)

The Third Department of Internal Medicine, Chiba University School of Medicine, Chiba, Japan

Mailing address: Katsuhiro Ono, MD, The Third Department of Internal Medicine, Chiba University School of Medicine, 1-7-1 Inohana, Chuuou-ku, Chiba City, Chiba 260-8670, Japan

\section{Methods}

\section{Subjects}

Thirty-seven subjects with hyperlipidemia (HL), hypertension (HT), and/or diabetes mellitus (DM) were studied by MRI ( 19 men with a mean age of $62.9 \pm 10.0$ years; 18 women aged $62.9 \pm 10.6$ years). Each gave informed consent. Hyperlipidemia, defined as a serum total cholesterol level $>220 \mathrm{mg} / \mathrm{dl}$ was present in 30 subjects ( $15 \mathrm{men})$, HT in 28 cases (14 men), and DM in 14 cases (10 men) (Table 1). Two patients had cerebral infarction associated with carotid stenosis.

\section{MRI}

MRI (Signa Horizon 1.5 T ver 5.7, GE Yokokawa Medical Co, Tokyo, Japan) was performed with the following pulse sequences: 2D-TOF images: repetition time (TR): $20 \mathrm{~ms}$, echo time (TE): $5 \mathrm{~ms}$, flip angle: $60^{\circ}$, slice thickness: $3 \mathrm{~mm}$, field of view (FOV): $20 \times 20 \mathrm{~cm}$, cardiac gating; T1-weighted SE images: TR: gating (800-1,200 ms), TE: $23 \mathrm{~ms}$, slice thickness: $3 \mathrm{~mm}$, FOV: $12 \times 12 \mathrm{~cm}$, matrix: $256 \times 192$, cardiac gating; T2-weighted SE images: TR: gating $(2,100-3,800$ $\mathrm{ms}$ ), TE: $80 \mathrm{~ms}$, slice thickness: $3 \mathrm{~mm}$, FOV: $12 \times 12 \mathrm{~cm}$, matrix: $256 \times 192$, cardiac gating; proton density (PD) SE images: TR: gating $(2,100-3,800 \mathrm{~ms})$, TE: $23 \mathrm{~ms}$, slice thickness: $3 \mathrm{~mm}$, FOV: $12 \times 12 \mathrm{~cm}$, matrix: $256 \times 192$. Breath hold-

Table 1 Cardiovascular Risk Factors of the Study Subjects

\begin{tabular}{lcccc}
\hline \hline \multirow{2}{*}{ Subjects } & \multicolumn{4}{c}{ Riskfactors } \\
\cline { 2 - 5 } & $D M$ & $H L$ & $H T$ & None \\
\hline $\begin{array}{l}19 \text { men } \\
(63.1 \pm 9.9 \text { years old })\end{array}$ & 10 & 15 & 14 & 2 \\
$\begin{array}{l}18 \text { women } \\
(62.9 \pm 10.6 \text { years old })\end{array}$ & 4 & 15 & 14 & 1 \\
\hline
\end{tabular}

DM, diabetes mellitus; HL, hyperlipidemia; HT, hypertension. 
ing was not used. Axial 2D-TOF images were obtained from the origin of the carotid arteries to $5 \mathrm{~cm}$ above the carotid bifurcation. We regarded a low signal intensity area inside the arterial wall on 2D-TOF as atheroma and examined the features of those that agreed with the findings on ultrasonography or SE images. We defined a low signal intensity area in the center of the vessel lumen as turbulent flow. The signal intensity of atheroma on SE images was classified as follows: high intensity (equal to subcutaneous tissue), slightly high intensity (between high and isointense), isointense (equal to muscle), and low intensity (lower than muscle). Five observers read the MRI before ultrasonography. Assessment of the atheroma was decided by majority. All observers agreed as to detecting atheroma on 2D-TOF. We disagreed on grading the signal intensity of atheroma in a few cases, but we agreed on the definition of 'high' signal intensity.

\section{Ultrasonography}

Carotid ultrasonography was done within 3 weeks of MRI. B-mode and color Doppler images were obtained with an SSD-2000 (Aloka Co) and a 7.5-MHz probe. The longand short-axis images were used to measure the thickness

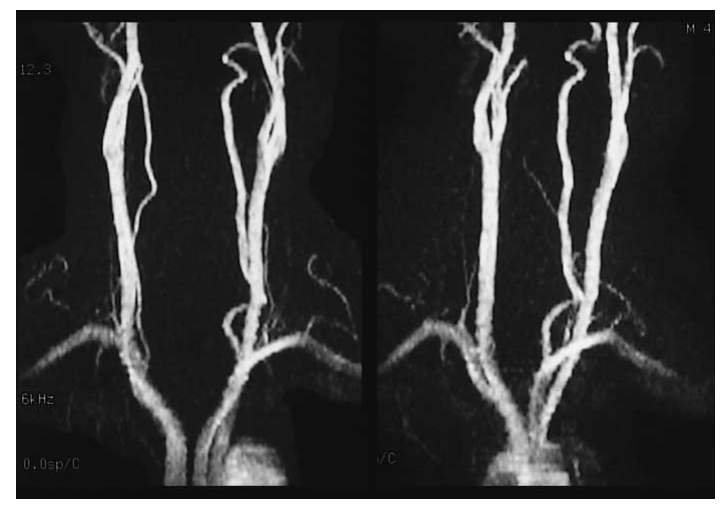

Fig 1. 2-D gated TOF MR image showing normal carotid arteries in a 73-year-old man with hyperlipidemia but without atheroma. of the intima-media complex by $0.1 \mathrm{~mm}$; thickness greater than $1.1 \mathrm{~mm}$ were regarded as atheroma. ${ }^{0}$

\section{Results}

\section{D-TOF Images}

Axial images of both the common carotid, internal carotid, external carotid, and vertebral arteries were obtained and the reconstructed 3-dimensional (D) images were clear in all 37 cases. Fig 1 shows the carotid arteries of a 73-yearold man with hyperlipidemia but without atheroma.

Atheroma was detected in $23(65 \%)$ of the 37 patients in the right common carotid $(n=11)$, right internal carotid $(n=9)$, left common carotid $(n=12)$, left internal carotid $(n=16)$, and left external carotid $(n=1)$. The most common site for atheroma was the carotid bifurcation, particularly from the posterior wall of the common carotid to the internal carotid (Table 2). Fig 2A is a reconstructed 2D-TOF image from a 42-year-old man with no other risk factors than smoking 40 cigarettes per day. Large atheroma were found at both carotid bifurcations. The right internal carotid was distended near the bifurcation and showed a slightly low signal intensity on the frontal image. No intense projection was found at the origin of left internal carotid on the left anterior oblique image $\left(30^{\circ}\right)$. On axial images obtained at the level of the bifurcation (Fig 2B), large atheroma with a low signal intensity were detected in the right and left internal carotid. Local dilation was seen at the site of large atheroma on 2DTOF images, suggesting vascular remodeling, and this change was found in all subjects with atheroma. However, turbulent flow around the bifurcation interfered with the quality of the images. Fig 3 shows follow-up 2D-TOF images from the case shown in Fig 2 after 1 year obtained

Table 2 The Locations of Atheroma on 2D-TOF Images

\begin{tabular}{lrrc}
\hline \hline & $C C$ & $I C$ & $E C$ \\
\hline Right & 11 & 9 & 0 \\
Left & 12 & 16 & 1 \\
\hline
\end{tabular}

$C C$, common carotid; IC, internal carotid; EC, external carotid.
A

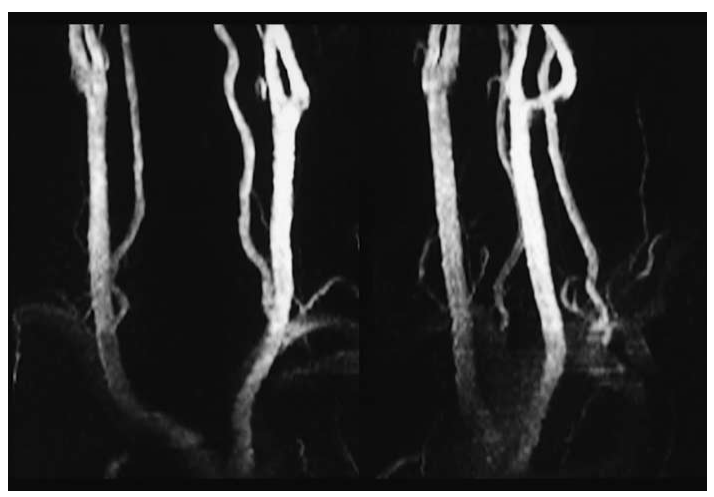

Fig 2. (A) 2D-TOF image from a 42-year-old man with only the risk factor of smoking 40 cigarettes per day. Atheroma can be seen on the posterior wall of the right and left carotid bifurcations. (Left) Frontal image. (Right) Left anterior oblique image $\left(30^{\circ}\right)$. (B) Axial images from the same patient.
B

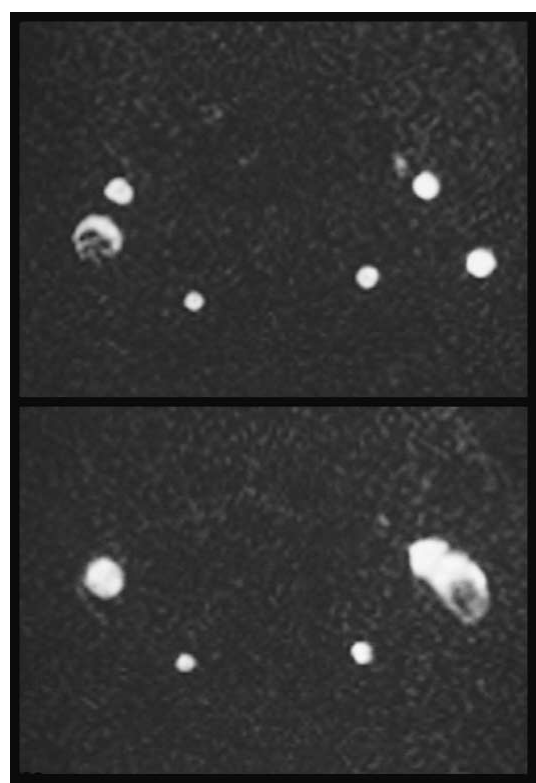


A

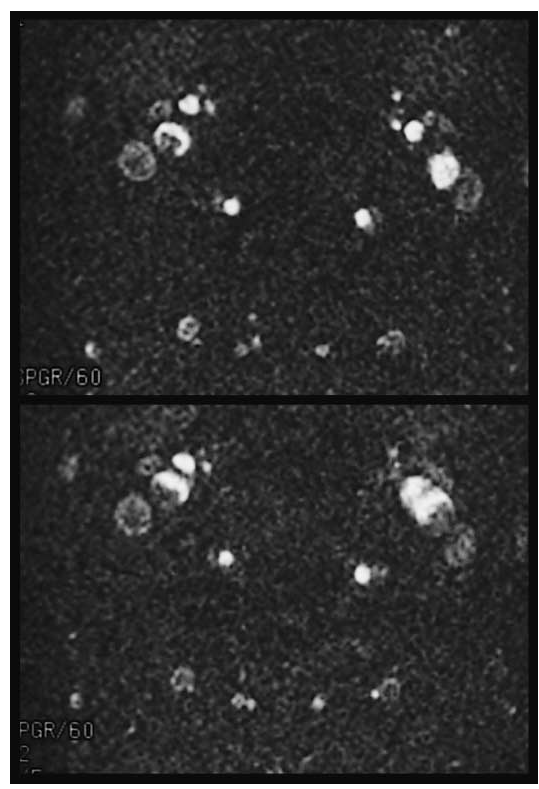

B

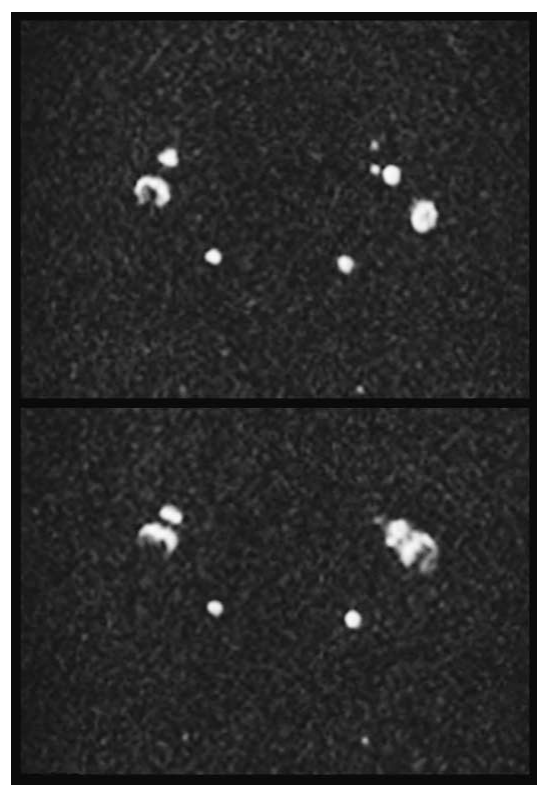

Fig 3. (A) A 2D-TOF image of the same case as Fig 2 performed after the injection of contrast medium (gadolinium-DTPA). (B) Corresponding conventional 2D-TOF image. before and after injection of contrast medium. After injection of contrast, the low intensity area was imaged in the same way as in conventional 2D-TOF images, confirming that it was not an artifact, but atheroma. No atheroma was detected in 13 subjects. Other findings on MRI included obstruction of the left vertebral artery and the left subclavian artery in one subject each.

MRA images were compared with the ultrasound findings in 34 subjects. The site of atheroma detected by 2D-TOF imaging agreed with the site on ultrasound in 23 of 24 subjects with low-intensity areas on MRI. MRI agreed with the ultrasonography findings in a total of 33 subjects, including 10 without atheroma. When compared with ultrasonography, 2D-TOF images showed 97\% agreement (Table 3). Fig 4 shows a 72-year-old man with hyperlipidemia, hypertension, and diabetes. There were low signal intensity areas in the left carotid bifurcation and obstruction of the right
Table 3 Findings on Magnetic Resonance Imaging (MRI) and Ultrasonography

\begin{tabular}{lcc}
\hline \hline & Ultrasound $(+)$ & Ultrasound $(-)$ \\
\hline$M R I(+)$ & 23 & 1 \\
$M R I(-)$ & 0 & 10 \\
\hline
\end{tabular}

$(+)$, atheroma was detected; (-), no atheroma. $N=34,97 \%$ agreement.

common carotid (Fig 4A). Digital subtraction angiography (DSA) also showed the same findings (Fig 4B), and atheroma was detected at the same site by ultrasonography (Fig 4C).

\section{Spin-Echo Images}

It is possible to identify different components based on variations of the signal intensity on T1-weighted SE (T1-SE), T2-weighted SE (T2-SE) and proton density SE (PD-SE)
A

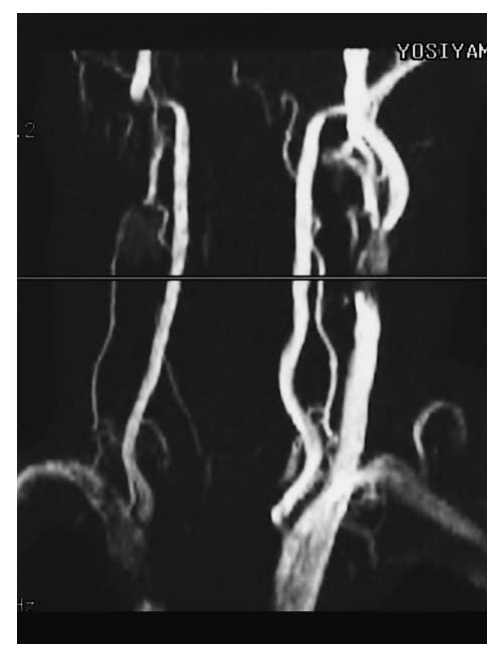

B

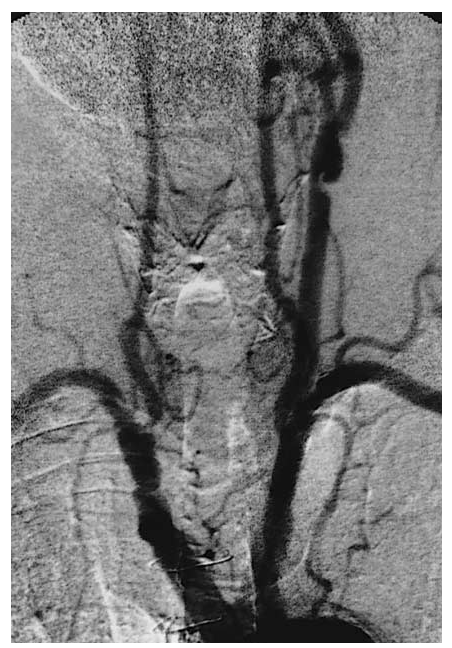

C

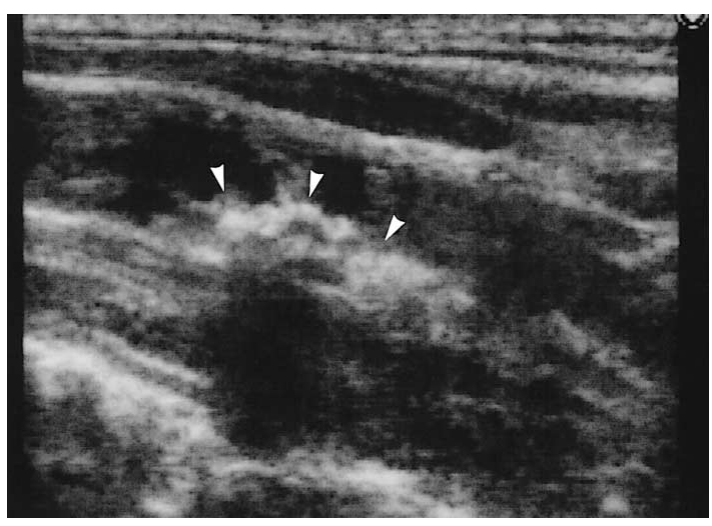

Fig 4. (A) 2D-TOF image from a 72-year-old man with all risk factors. There is stenosis of the left carotid bifurcation and obstruction of the right common carotid. (B) The same findings are seen on DSA. (C) Ultrasonography shows atheroma at the same site. 
Table 4 Findings on Spin-Echo (SE) Images

\begin{tabular}{|c|c|c|c|c|c|c|c|c|c|}
\hline \multirow{3}{*}{ Case no. } & \multirow{3}{*}{ Age/Sex } & \multicolumn{8}{|c|}{ Location of Atheroma } \\
\hline & & \multicolumn{3}{|c|}{ Risk factor } & \multirow{2}{*}{$2 D-T O F$} & \multirow{2}{*}{$S E$} & \multicolumn{3}{|c|}{ SE images } \\
\hline & & $H L$ & $H T$ & $D M$ & & & $T 1$ & $T 2$ & $P D$ \\
\hline 1 & $48 M$ & + & + & + & $L$ & $I$ & iso/sl.high/high & low/high/sl.high & \\
\hline 2 & $60 M$ & + & + & + & $R$ & $C$ & low/high & low/high & \\
\hline 3 & $72 M$ & + & + & + & $R$ & $C I$ & sl.high & sl.high/high & high \\
\hline \multirow[t]{2}{*}{4} & $74 M$ & + & + & + & $R$ & $C I$ & low/high & iso & high/low \\
\hline & & & & & $L$ & $C I$ & low/iso/sl.high & iso & high/low \\
\hline 5 & $66 F$ & + & + & + & $L$ & $I$ & low/iso & iso & iso \\
\hline 6 & $61 M$ & + & + & + & $L$ & $I$ & high/low & iso & \\
\hline 7 & $63 F$ & + & + & + & $R$ & $I$ & low/high & sl.high/low & \\
\hline 8 & $73 F$ & + & + & + & $L$ & $C I$ & low & low/iso & \\
\hline 9 & $65 F$ & + & + & + & $R$ & $I$ & sl.high & iso & \\
\hline 10 & $78 F$ & + & + & - & $L$ & $C$ & high/low & iso & \\
\hline 11 & $71 M$ & + & + & - & $L$ & $C I$ & low/high & high & \\
\hline 12 & $51 F$ & + & + & - & $L$ & $C$ & high & high & high \\
\hline 13 & $68 M$ & + & + & - & $L$ & $C$ & iso/low/high & low/high & iso/high \\
\hline 14 & $49 M$ & + & + & - & $R$ & $C$ & low & & \\
\hline \multirow[t]{2}{*}{15} & $59 F$ & + & + & - & $R$ & $C I$ & iso & iso & low \\
\hline & & & & & $L$ & $C I$ & iso/high/low & iso/high & high \\
\hline 16 & $65 M$ & + & - & + & $R$ & $I$ & high & iso/low/sl.high & \\
\hline 17 & $55 F$ & + & - & - & $R$ & $I$ & iso & iso & high \\
\hline 18 & $62 F$ & + & - & - & $L$ & $I$ & high & high & \\
\hline 19 & $63 F$ & + & - & - & $R$ & $I$ & low/sl.high & & low/sl.high \\
\hline 20 & $60 M$ & - & + & + & $L$ & $C$ & low/sl.high & & \\
\hline 21 & $72 M$ & - & + & - & $L$ & $I$ & iso/low & sl.high & low \\
\hline 22 & $69 M$ & - & - & - & $R$ & $I$ & iso & high & \\
\hline 23 & $43 M$ & - & - & - & $R$ & $C I$ & low & iso & iso \\
\hline
\end{tabular}

HL, hyperlipidemia; HT, hypertension; DM, diabetes mellitus; $R$, right; L, left; $C$, common carotid; $I$, internal carotid. Cases 22 and 23 were heavy smokers.

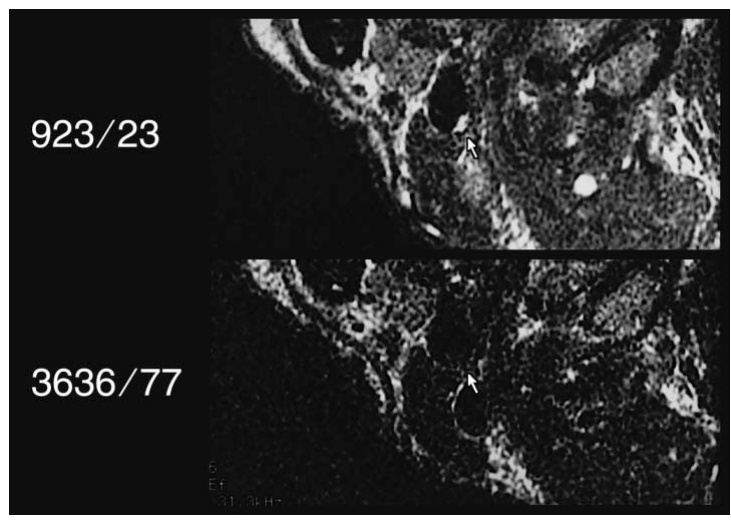

Fig 5. T1-SE image (Upper) from a 65-year-old man with hyperlipidemia and diabetes mellitus showing a lesion (arrow) with a high signal that was low on T2-SE (Lower).

images ${ }^{7-9}$ Lipid components show a very high signal intensity on T1-SE images, a low signal intensity on T2-SE images, and a high signal intensity in PD-SE images. Thus, a high signal intensity on T1-SE images is characteristic of lipid, whereas a high signal intensity on T2-SE images is characteristic of fibrous tissue. SE images were obtained in 21 subjects with atheroma and 2 subjects with carotid wall thickening. T1-SE images showed a high signal intensity characteristic of lipid components in 12 of 19 subjects with HL (63\%), 0 of 4 subjects without HL (0\%) and 10 of 17 subjects with HT (59\%), who also all had HL. And 2 of 6 subjects without HT (33\%), 6 of 11 subjects with DM $(55 \%)$, and 6 of 12 subjects without DM $(50 \%)$ showed a high signal intensity on T1-SE. Thus, a high signal intensity

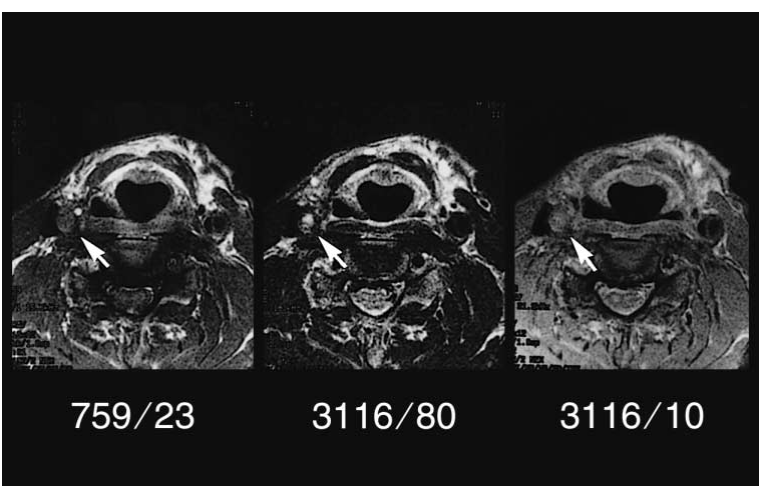

Fig 6. T2-SE image (Middle) from a 72-year-old man with hyperlipidemia, hypertension and diabetes showing a high signal intensity lesion (arrow) that was not as high on the T1-SE (Left) and PD-SE (Right) images.

on T1-SE images was common in subjects with HL and/or HT, suggesting that accumulation of lipids in atheroma was related to HL and HT, particularly HL. In contrast, a high signal intensity on T2-SE (characteristic of fibrous tissue) showed no relationship to these cardiovascular risk factors. Fig 5 shows a T1-SE image from a 65-year-old man with $\mathrm{HL}$ and DM, which has a high signal intensity area that was low signal intensity on T2-SE images, findings that are compatible with a lipid component of atheroma. Fig 6 is a T2-SE image from a 72-year-old man with all 3 risk factors, showing the right common carotid obstructed by a high signal intensity lesion. The obstruction had a lower signal on T1-SE and PD-SE images, suggesting that the atheroma had a fibrous component. 


\section{Discussion}

In the present study, clear axial images of the bilateral common carotid, internal carotid, external carotid, and vertebral arteries, as well as reconstructed 3D images, were obtained in all subjects. The pulse sequences used were decided on the basis of previous reports. ${ }^{7}$ Image quality was sometimes reduced by turbulent flow.

For evaluation of the severity of stenosis, the accuracy of gadolinium DTPA-enhanced MRI is reported to be as high as that of DSA 10 The present study showed that $2 \mathrm{D}-\mathrm{TOF}$ imaging without contrast is also useful for the detection of atheroma. Almost all of the atheroma were detected around the carotid bifurcation and near the origin of the internal carotid rather than in the external carotid, which may be because of the kink at the origin of the internal carotid. Lowsignal-intensity artifacts were mainly found near the internal carotid origin, probably because the direction of flow at this site is perpendicular to the detector, so the signal is weaker, and because the kink in the artery causes turbulent flow. Turbulent flow can promote thrombus formation. In this study, comparing the right and left carotid arteries on axial images allowed us to estimate the extent of vascular remodeling. Yasui et al first reported that remodeling of the carotid arteries was one of the causes of disagreement between DSA and ultrasound findings ${ }^{11}$ MRI can show the right and the left common carotid arteries simultaneously and thus is useful for estimation of remodeling. There have been some reports about the relationship between vascular remodeling and ultrasound findings, and about the relation between remodeling and the type of artery ${ }^{1-15}$ However, studies of the relationship between remodeling and MRI findings have not been performed. When deciding whether a low signal intensity area was caused by atheroma or an artifact, the most important point is whether or not the low signal intensity area is inside the arterial wall. On this basis, we defined a low signal intensity area in the center of the vessel lumen as turbulent flow. Another important point is whether vascular remodeling is present or not.

Angiography is the gold standard imaging technique for diagnosing arterial stenosis, but it has been reported that the detection of carotid stenosis on 3D-TOF images agrees with that seen on DSA, and that MRA and ultrasound can be used to decide the indications for endarterectomy? We regarded the findings on $2 \mathrm{D}-\mathrm{TOF}$ that agreed with those on ultrasound as atheroma, and obtained similar SE images. Compared with MRI, ultrasound is convenient, but its field of examination is limited to around the bifurcation. In the present study, we could observe a large area from the origin of the carotid arteries to $5 \mathrm{~cm}$ above the bifurcation using MRI. Also, ultrasound will not show obstructed vertebral or subclavian arteries, unlike MRI. Furthermore, ultrasound only shows lesions as echolucent or bright, but MRI allows more subtle gradations of signal intensity so that the components of atheroma can be predicted. Not only the extent of stenosis, but also the features of the atheroma are important in determining the risk of clinical events. In this regard, 2DTOF is useful for evaluating stenosis of the carotid arteries and SE images are useful for detecting the features of the atheroma.

We found that T1-SE, T2-SE, and PD-SE images were all useful for characterization of atheroma. Atheroma in the aorta has been detected by SE images in animal studies, ${ }^{16-19}$ and atheroma in human arteries has also been characterized in vitro ${ }^{20-24}$ Yuan et al reported that they measured carotid atheroma in vivo using MRI 25 and Toussaint et al reported that MRI findings of the carotid artery agreed with the histology after endarterectomy? In their study, the spatial resolution was $0.39 \mathrm{~mm}$ and they could detect the components of atheroma.

In the present study, we obtained equivalent resolution to Toussaint et al. We divided the signal intensity into 4 grades (high, slightly high, isointense, and low). There have been reports $^{7-9}$ on the signal intensity of atheroma components (ie, lipid, fibrin and calcification) in which MR images were compared with histology. In the present study we could detect different components based on the signal intensity in T1-SE, T2-SE, and PD-SE images. Lipid shows a high signal intensity in T1-SE and PD-SE images, and with these images such a signal does not occur other atheroma components, so it is characteristic of lipid. Similarly, a higher signal than lipid on T2-SE is indicative of fibrous tissue, whereas a low signal on T1-SE and PD-SE images indicates calcification. We found a relationship between the signal intensity of atheroma and risk factors. Atheroma showing a high signal on T1-SE were common in subjects with HL and HT, and all subjects with DM or HT who showed such a signal also had HL, which suggests that lipid-rich atheroma is related to HL and HT, particularly HL. In contrast, a relationship between fibrous tissue in atheroma and these risk factors was not found. We also need to investigate calcification and bleeding in atheroma, but computed tomography may be more suitable to detect calcification than MRI. A study of the relationship between the features of atheroma on SE images and the occurrence of cerebral infarction may also be worthwhile.

\section{References}

1. Davis MJ, Richardson PD, Woolf N, Katz DR, Mann J: Risk of thrombosis in human atherosclerosis plaque: Role of extracellular lipid, macrophage, and smooth muscle cell content. Br Heart J 1993; 69: 377-381

2. Fuster V, Stein B, Ambrose JA, Badimion L, Badimonn JJ, Chesebro $\mathrm{JH}$ : Atherosclerotic plaque rupture and thrombosis: Evolving concepts. Circulation 1990; 82: 1147-1159

3. Richardson PD, Davis MJ, Born GV: Influence of plaque configuration and stress distribution on fissuring of coronary atherosclerotic plaques (see comments). Lancet 1989; 2: $941-944$

4. Rasanen HT, Manninen HI, Vanninen RL, Vainio P, Berg M, Saari T, et al: Mild carotid artery atherosclerosis Assessment by 3-dimensional time-of- flight magnetic resonance angiography, with reference to intravascular ultrasound imaging and contrast angiography. Stroke 1999; 30: $827-833$

5. Huston J, Nichols DA, Luetmer PH, Rydberg CH, Lewis BD, Meyer FB, et al: MR angiographic and sonographic indications for endarterectomy. Am J Neuroradiol 1998; 19: 309-315

6. Carriero A, Scarabino T, Magarelli N, Marano R, Ambrosini R, Salvolini U, et al: High-resolution magnetic resonance angiography of the internal carotid artery: 2D vs 3D TOF in stenotic disease. Eur Radiol 1998; 8: 1370-1372

7. Ingersleben G, Schmiedl UP, Hatsukami TS, Nelson JA, Subramaniam DS, Furguson MS, et al: Characterization of atherosclerosis plaques at the carotid bifurcation: Correlation of MR imaging with histoloigic analysis: Preliminary study. Radiographics 1997; 17: 1417-1423

8. Toussaint JF, LaMuraglia GM, Southern JF, Fuster V, Kantor HL: Magnetic resonance images lipid, fibrous, calcified, hemorrhagic, and thrombotic components of human atherosclerosis in vivo. Circulation 1996; 94: $932-938$

9. Tenjin H, Yasukochi K, Nakahara Y, Higuchi T, Naruse S, Ueda S, et al: Preoperative detection of the composition of atherosclerotic plaque in the carotid artery using ultrasonography and magnetic resonance imaging. Neurol Med Chir 1996; 37: 505-511

10. Tell GS, Howard G, McKinney WM: Risk factors for site specific extracranial carotid artery plaque distribution as measured by B-mode ultrasound. J Clin Epidemiol 1989; 42: 551-559

11. Remonda L, Heid O, Schroth G: Carotid artery stenosis, occlusion, and 
pseudo-occlusion: First-pass, gadolinium-enhanced, three-dimensional MR angiography: Preliminary study. Radiology 1998; 209: 95-102

12. Yasui K, Watanabe H, Abe Y, Hakusui S, Ando T, Yanagi T: A comparison of ultrasonography and cerebral angiography for the evaluation of extracranial carotid stenosis. Clin Neurol 1997; 37: 783-789

13. Pasterkamp G, Schoneveld AH, Wolferen W, Hillen B, Clarijs RJG, Haudenschild CC, et al: The impact of atherosclerotic arterial remodeling on percentage of luminal stenosis varies widely within the arterial system: A postmortem study. Arterioscler Thromb Vasc Biol 1997; 17: 3057-3063

14. Labropoulos N, Zarae J, Mansour A, Kang SS, Baker WH: Compensatory arterial enlargement is a common pathobiologic response in early atherosclerosis. Am J Surg 1998; 176: $140-143$

15. Kiechl S, Willeit J: The natural course of atherosclerosis. Part I: Incidence and progression. Arterioscler Thromb Vasc Biol 1999; 19: $1484-1490$

16. Kiechl S, Willeit J: The natural course of atherosclerosis. Part II: Vascular remodeling. Arterioscler Thromb Vasc Biol 1999; 19: 14911498

17. Skinner MP, Yuan C, Mitsumori L, Hayes CE, Raines EW, Nelson JA, et al: Serial magnetic resonance imaging of experimental atherosclerosis detects lesion fine structure, progression and complication in vivo. Nat Med 1995; 1: 69-73

18. Yuan C, Skinner MP, Kaneko E, Mitsumori ML, Hayes CE, Raines EW, et al: Magnetic Resonance imaging to study lesions of atherosclerosis in the hyperlipidemic rabbit aorta. Magn Reson Imaging 1996;
14: $93-102$

19. Ford JC, Shalansky-Goldberg RD, Golden M: MR microscopy of the arterial wall in an experimental model of atherosclerosis: Preliminary results. J Vasc Interv Radiol 1997; 8: $93-99$

20. Manninen HI, Vanninen RL, Laitinen M, Rasanen H, Vainio P, Luoma JS, et al: Intravascular ultrasound and magnetic resonance imaging in the assessment of atherosclerotic lesions in rabbit aorta: Correlation to histopathologic findings. Invest Radiol 1998; 33: 464-471

21. Gorter M, Goldmann A, Mohr W, Widder B: Tissue characterization of atherosclerotic carotid plaques by MRI. Neuroradiology 1995; 37: 631-635

22. Martin AJ, Gotlieb AI, Henkelman RM: High-resolution MR imaging of human arteries. J Magn Reson Imaging 1995; 5: 93-100

23. Toussaint JF, Southern JF, Fuster V, Kantor HL: T2-weighted contrast for NMR characterization of human atherosclerosis. Arterioscler Thromb Vasc Biol 1995; 15: 1533-1542

24. Halliburton SS, Paschal CB: Atherosclerotic plaque components in human aortas contrasted by ex vivo imaging using fast spin-echo magnetic resonance imaging and spiral computed tomography. Invest Radiol 1996; 31: 724-728

25. Martin AJ, Ryan LK, Gotlieb AI, Henkelman RM, Foster FS: Arterial imaging: Comparison of high-resolution US and MR imaging with histologic correlation. Radiographics 1997; 17: 189-202

26. Yuan C, Beach KW, Smith LH Jr, Hatsukami TS: Measurement of atherosclerotic carotid plaque size in vivo using high resolution magnetic resonance imaging. Circulation 1998; 98: 2666-2671 